\title{
High-Density Dynamics of Laser Wakefield Acceleration from Gas Plasmas to Nanotubes
}

\author{
Bradley Scott Nicks ${ }^{1, *} \mathbb{\infty}$, Ernesto Barraza-Valdez ${ }^{1}$, Sahel Hakimi ${ }^{1}{ }^{\circledR}$, Kyle Chesnut ${ }^{1}{ }^{1}$, \\ Genevieve DeGrandchamp ${ }^{1}$ (), Kenneth Gage ${ }^{1}{ }^{1}$, David Housley ${ }^{2}$, Gregory Huxtable ${ }^{1}$, Gerard Lawler $^{3}{ }^{(1)}$, \\ Daniel Lin ${ }^{1}$, Pratik Manwani ${ }^{3}{ }^{1}$, Eric Nelson ${ }^{1}$, Gabriel Player ${ }^{1}$, Michael Seggebruch ${ }^{1}$, James Sweeney ${ }^{1}$, \\ Joshua Tanner ${ }^{1}$, Kurt Thompson ${ }^{2}$ and Toshiki Tajima ${ }^{1}$
}

1 Department of Physics and Astronomy, University of California, Irvine, CA 92697, USA; ernestob@uci.edu (E.B.-V.); sahelh@uci.edu (S.H.); kdchesnu@uci.edu (K.C.); gdegrand@uci.edu (G.D.); gagek@uci.edu (K.G.); huxtablg@uci.edu (G.H.); djlin1@uci.edu (D.L.); ecnelson@uci.edu (E.N.); gplayer@uci.edu (G.P.); mseggebr@uci.edu (M.S.); jmsweene@gmail.com (J.S.); jetanner@uci.edu (J.T.); ttajima@uci.edu (T.T.)

2 Department of Physics, University of California, San Diego, CA 92093, USA; dhousley@eng.ucsd.edu (D.H.); k6thomps@ucsd.edu (K.T.)

3 Department of Physics and Astronomy, University of California, Los Angeles, CA 90095, USA; gelawler@physics.ucla.edu (G.L.); pkmanwani@gmail.com (P.M.)

* Correspondence: bnicks@uci.edu

check for updates

Citation: Nicks, B.S.; Barraza-Valdez,

E.; Hakimi, S.; Chesnut, K.;

DeGrandchamp, G.; Gage, K.;

Housley, D.; Huxtable, G.; Lawler, G.;

Lin, D.; et al. High-Density Dynamics

of Laser Wakefield Acceleration from

Gas Plasmas to Nanotubes. Photonics

2021, 8, 216. https://doi.org/

$10.3390 /$ photonics 8060216

Received: 10 May 2021

Accepted: 8 June 2021

Published: 11 June 2021

Publisher's Note: MDPI stays neutral with regard to jurisdictional claims in published maps and institutional affiliations.

Copyright: (c) 2021 by the authors. Licensee MDPI, Basel, Switzerland. This article is an open access article distributed under the terms and conditions of the Creative Commons Attribution (CC BY) license (https:// creativecommons.org/licenses/by/ $4.0 /)$.

\begin{abstract}
The electron dynamics of laser wakefield acceleration (LWFA) is examined in the highdensity regime using particle-in-cell simulations. These simulations model the electron source as a target of carbon nanotubes. Carbon nanotubes readily allow access to near-critical densities and may have other advantageous properties for potential medical applications of electron acceleration. In the near-critical density regime, electrons are accelerated by the ponderomotive force followed by the electron sheath formation, resulting in a flow of bulk electrons. This behavior represents a qualitatively distinct regime from that of low-density LWFA. A quantitative entropy index for differentiating these regimes is proposed. The dependence of accelerated electron energy on laser amplitude is also examined. For the majority of this study, the laser propagates along the axis of the target of carbon nanotubes in a 1D geometry. After the fundamental high-density physics is established, an alternative, 2D scheme of laser acceleration of electrons using carbon nanotubes is considered.
\end{abstract}

Keywords: laser-wakefield acceleration; critical-density plasmas; oncology; electron beams; carbon nanotubes; sheath acceleration; entropy

\section{Introduction}

Laser Wakefield Acceleration (LWFA) [1] is a compact method to accelerate charged particles to high energies that was first purposed by Tajima and Dawson [2] in 1979. While the accelerating electric field in a conventional linear accelerator is limited by the breakdown threshold of its device walls, the inherently broken-down nature of plasma allows plasma-based accelerators to access far higher electric fields. Consequently, plasmabased accelerators can access far higher accelerating gradients than those available to conventional accelerators, reaching potentially $\mathrm{GeV}$ per $\mathrm{cm}$ or higher. The development of Chirped Pulse Amplification (CPA) [3] allowed experimental access to the high laser intensities originally proposed for LWFA $\left(10^{18} \mathrm{~W} / \mathrm{cm}^{2}\right)$, and LWFA was experimentally verified shortly thereafter $[4,5]$. Since then, many experiments have demonstrated this technique in different regimes, and the field has grown steadily.

Accelerators have many applications in our current society, one of the most important being their use in radiation therapy. Energetic beams composed of constituents such as of $X$-rays, electrons, or protons can be used to treat cancer. These energetic beams can ionize 
molecules and thus damage cellular DNA. Cells with damaged DNA can not reproduce and are eliminated through natural processes in the body. The type of beam used depends highly on the size and location of cancer being treated. For example, while X-rays and electrons deposit most of their energy in the surface layers, protons can be controlled to deposit their energy at a specific depth due to Bragg peak phenomenon [6,7], dramatically reducing damage to healthy cells. Proton therapy contends with other limitations, however [8]. For this study we are interested in electron beams, which can have shallow or deep penetration depths depending on energy.

Current accelerators used for radiation therapy use traditional linear accelerator technology. To produce an electron or X-ray beam, electrons from an electron gun are accelerated and guided through waveguides using electric fields and magnets. These electrons can eventually hit a target and produce X-rays. However, the material breakdown limits of linear accelerators tend to require that these machines be large and costly. The typical electron energy needed for radiation therapy is between 5 and $20 \mathrm{MeV}$. LWFA techniques can accelerate electrons to these energies in length scales between microns and millimeters. Such a high acceleration gradient reduces the size and cost of these machines, consequently increasing their availability.

Research in the use of LWFA to generate electron beams for medical applications has presently proceeded for more than two decades. Initially, these efforts focused on the generation of high-quality electron beams with energies roughly in the range of 6-25 MeV, as would be applicable for conventional, external sources of radiation for cancer therapy [9-15]. Recent innovations in the field of fiber lasers has produced a critical advancement for this effort: the Coherent Amplification Network (CAN) [16], in which many individual micron-scale fiber lasers are coherently combined and amplified to provide both high repetition rate and high power. In such a scheme, laser accelerators could possibly be further compactified as to be viable even for endoscopic applications. If electrons can be generated inside of a patient's body (or in an intraoperative [12,13] fashion), the desired energy of these accelerated electrons (and X-rays converted from them) is much lower than those of typical, high-energy LWFA ( $\mathrm{MeV}$ and above), as they need not traverse healthy tissues before reaching the tissues to be treated.

In LWFA a clear path to the desired MeV-range electrons exists through utilizing high plasma densities. Consequently, we wish to study LWFA in the high-density regime. This regime has been less explored in detail than that of the more typical, low-density regime of LWFA. To establish a conceptual footing in this less understood regime, we exert our main focus on the fundamental physics of high-density electron acceleration. In doing so, we revive and take advantage of past work on sheath acceleration [17]. To this end, robust laser-plasma interaction is desired, and so we employ higher laser intensities than would likely be possible to achieve in a medical application based on fiber lasers, though CAN techniques may ultimately mitigate such concerns. Previous work [18] treated the practical considerations of fiber lasers and medical applications more explicitly.

Generally, gas plasmas are used as the electron source in LWFA. Indeed, as is described in more detail in Section 2, low-density plasmas are ideal for typical LWFA experiments. For medical applications, however, particularly for cases where the electron source is brought inside the body, a solid-state electron source is much more desirable for two chief reasons. First, as described above, the electrons produced from such a medium are of a desirably low energy. Second, a solid-state electron source potentially avoids the creation of gas plasma and the necessity of maintaining a vacuum, both of which are highly undesirable for endoscopic medical applications.

One possible electron source satisfying these requirements may be provided by an arrangement of carbon nanotubes (CNTs) such as those in Figure 1, which allows access to much high densities than those easily attained by gas plasmas. Various other advantageous properties of CNTs motivate such a scheme. Carbon nanotubes can be synthesized to have metallic properties by using the armchair configuration $[19,20]$. Though CNTs alone generally can have a lower conductivity than that of metals, the conductivity of 
composite materials such as CNTs embedded in copper nanotubes can reach or exceed that of metals [21,22]. Additionally, CNTs have been shown to have electron carrier mobility much higher than that of metals [23]. For example, metals like aluminum and copper have mobilities of order $10^{1}$ to $10^{2} \mathrm{~cm}^{2} /(\mathrm{V} \cdot \mathrm{s})$ while single-wall CNTs can exhibit electron mobilities of order $10^{4} \mathrm{~cm}^{2} /(\mathrm{V} \cdot \mathrm{s})$. Other desirable properties of CNTs for the present study are a low work function (approximately $5 \mathrm{eV}$, similar to that of metals [24]) and an effective one-dimensional conductivity parallel to the CNT axis. We thus seek to assimilate our investigation of the physics of electron acceleration in the high-density regime to a possible CNT application. Specifically, the bulk of this work models electron acceleration from a laser propagating down the axis of a CNT bundle [? ], shown schematically in Figure 1b. Later, in Section 4, we consider an alternative arrangement of CNTs, shown in Figure 1b, that requires more advanced treatment.

The energy gain of electrons in LWFA is given by $\Delta \mathcal{E}=2 g\left(a_{0}\right) m_{e} c^{2}\left(n_{c} / n_{e}\right)$, where $a_{0}=e E_{0} / m_{e} \omega_{l} c$ is the normalized vector potential of the laser pulse with $E_{0}$ and $\omega_{l}$ representing the electric field and frequency of the laser. Here $e, m_{e}$, and $c$ are electron charge, electron mass, and speed of light, respectively. If the function $g\left(a_{0}\right)$ takes the form of the ponderomotive potential, then $g\left(a_{0}\right)=\sqrt{1+a_{0}^{2}}-1$ [26], but generally here $g\left(a_{0}\right)$ is considered to be of order unity at $a_{0}=1$. The electron energy gain is proportional to the ratio of $n_{c}$, the critical plasma density defined by the laser frequency, to $n_{e}$, the plasma or electron density. If the value of this ratio is near unity, the electron energy gain is on the order of $\mathrm{MeV}$ or lower. For this work we assume a common 800-nm Ti:Sapphire laser, which has a critical density of $n_{c} \approx 1.73 \times 10^{21} \mathrm{~cm}^{-3}$. In comparison, a quick estimate for the average electron density of a CNT with a radius of $10 \mathrm{~nm}$ gives $n_{e} \sim 10^{22} \mathrm{~cm}^{-3}$. As CNTs can flexibility possess radii both larger and smaller than that used in this estimation, CNTs readily allow access to both near- and super-critical densities as desired. In the following, we also study the scaling laws of electron energy gain $\Delta \mathcal{E}$ over the parameters of plasma density and intensity $a_{0}$ in addition to investigating the mechanics of electron acceleration in the high-density regime.

This work is divided as follows. Section 2 examines the physics of wakefield acceleration in the high-density regime by modeling propagation of a laser down the axis of a CNT bundle. Section 3 elaborates this topic by examining the accelerating potential of different regimes of laser amplitude. Building on these results, Section 4 explores the use of a an alternative arrangement of CNTs (see Figure 1) as a source of low-energy electrons such as might be useful to medical applications. Finally, Section 5 offers concluding remarks.

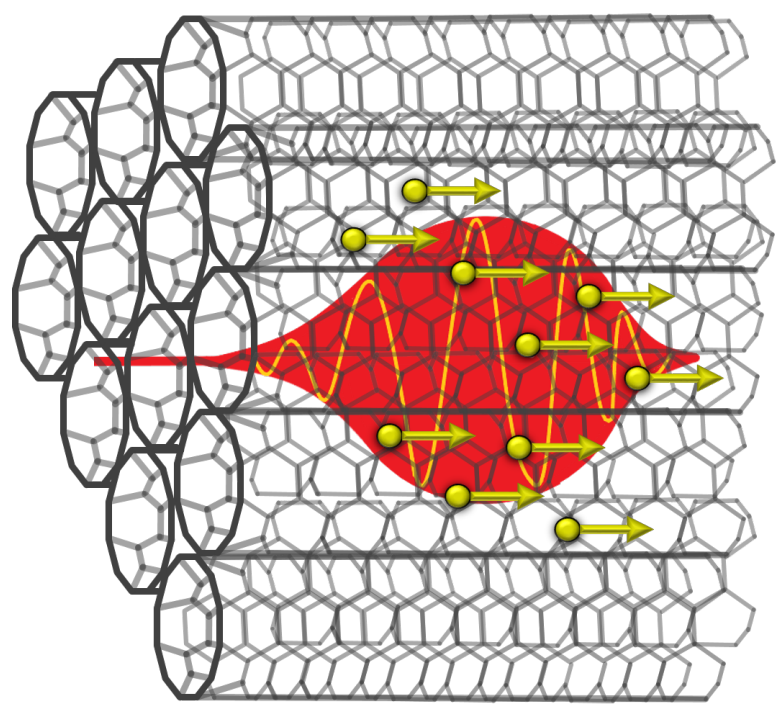

(a)

Figure 1. Cont. 


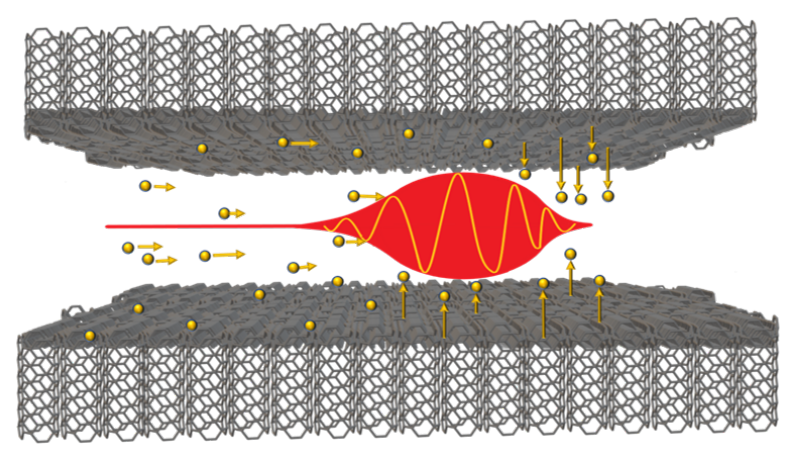

(b)

Figure 1. Illustration for proposed arrangement of carbon nanotubes to emulate high density plasma (not necessarily to scale). A laser pulse, shown in red, interacts with sheets of carbon nanotubes, shown in grey. The laser permeates the nanotubes, extracting and accelerating electrons, shown in yellow. Two arrangements are considered: (a) features CNTs arranged parallel to the laser propagation direction, and (b) features CNTs arranged perpendicularly to the laser propagation. The former case is treated for the majority of this work.

\section{Acceleration in the High-Density Regime}

If the plasma density is near the laser critical density, the typical physics of LWFA transitions into a qualitatively distinct regime where analytic extensions of conventional wakefield physics [27] may become insufficient. Crucially, as $n_{e}$ approaches $n_{c}$, the group velocity of the laser pulse, given by $v_{g}=c \sqrt{1-n_{e} / n_{\mathcal{c}}}$, approaches zero. The wake phase velocity is approximately equal to the laser group velocity and thus also approaches zero in this regime. The laser pump depletion and dephasing lengths, which are on the order of $L_{d} \sim L_{p} \sim \lambda_{p} a_{0}^{2}\left(n_{c} / n_{e}\right)$, also diminish with $v_{g}$ until they become shorter than the plasma wavelength $\lambda_{p}=2 \pi c / \omega_{p}$ for $a_{0}=1$. The length scale of laser-plasma interaction then becomes better described by the plasma skin depth $c / \omega_{p}$. As a consequence of the low laser group velocity $v_{g}$, the laser couples significantly to the bulk motion of the plasma, which is characterized by the plasma thermal velocity $v_{T}=\sqrt{T / m}$, where $T$ and $m$ here are most relevantly applied to electrons.

In contrast, wakefield physics in the low-density regime relies on a fast laser group velocity $\left(v_{g} \approx c \gg v_{T}\right)$. The laser penetrates deeply into the plasma without coupling to the bulk motion. The resulting wake phase velocity is then $v_{p h}=v_{g} \approx c \gg v_{T}$, and $L_{d} \sim L_{p} \gg \lambda_{p}$. This fast phase velocity and related long interaction length scale allow the laser to build a long and robust wakefield train. When electron injection occurs, the wakefield skims a small population of electrons from the bulk and accelerates them to high energies, unlike in the high-density case. This sharp divide in fundamental physics requires examination of the high-density regime on its own terms and a qualitative understanding apart from that of conventional wakefield acceleration.

The differences between the qualitative physics of the high- and low-density cases, which respectively represent waves of low and high phase velocity relative to the plasma thermal velocity, extends to many general features of plasma physics. For waves with $v_{p h} \sim v_{T}$, the wave couples to the bulk thermal motion of the plasma, typically producing macro-instabilities and turbulence. Plasma structures are thus fragile to such waves, and may disintegrate from wave-induced transport. In contrast, waves with $v_{p h} \gg v_{T}$ do not couple to the bulk thermal motion of the plasma, and the plasma and wave are insulated from each other. With regard to the wave fields, the wave can then reach a robust saturation amplitude before particle trapping begins to occur. In this limit, the wave trapping velocity [28] becomes approximately equal to the wave phase velocity, leading to the characteristic Tajima-Dawson saturation amplitude $E_{T D}=m \omega v_{p h} / q$, where $\omega$ is the wave frequency and $m$ and $q$ are respectively the mass and charge of the relevant species. At this saturation amplitude, wave-particle interaction manifests as the acceleration of 
a tail of extremely fast particles while the thermal distribution remains intact. Indeed, under the influence of the free energy of such a wave, plasma structures can be built rather than destroyed; the plasma is durable against the wave, rather than fragile. Because the original LWFA concept was built upon this high phase-velocity paradigm and departs from the sheath-forming, high-density regime [17], most present works on LWFA have avoided the high-density (low phase-velocity) regime. Thus it is the purpose of this work to first qualitatively distinguish these two regimes and then quantitatively characterize the differences in their most important dynamics. To do so, we isolate the longitudinal spatial dynamics and show their overwhelming influence on the departure of the physical characteristics of the above two regimes.

The dynamics of tsunami waves in different regimes of water depth provides a convenient analogy for these contrasting regimes of acceleration in high- and low-density plasmas. The deep water of the open ocean allows tsunami waves to propagate with a fast phase velocity given by $v_{p h}=\sqrt{g / k}$ with wavenumber $k$ [29], where $g$ is the constant of gravitational acceleration. This fast phase velocity causes only weak interaction with stationary objects (such as boats). Near the shore, however, the shallow water forces the wave to move with a slower phase velocity given by $v_{p h}=\sqrt{g h}$, where $h$ is the water depth. As its phase velocity slows, the wave steepens and amplifies until breaking occurs. Two key related consequences follow. First, the slow phase velocity causes strong and catastrophic coupling to stationary objects. Second, the turbulent process of wave breaking causes dredging of sediment from the seabed. The sediment is then incorporated into the wave and transported forwards, creating a visibly black wave. The momentum transport imparted to the sediment can be considered to represent an effective viscosity caused by the turbulence of wake breaking. In contrast, the wave in the open ocean does not incorporate sediment and thus remains blue.

The typical case of wakefield acceleration in a low-density $\left(n_{c} \gg n_{e}\right)$ plasma is analogous to that of the tsunami wave in the open ocean. The wakefield remains decoupled from the bulk electron population, leaving the wave ordered and "blue". In the case of highdensity $\left(n_{c} \sim n_{e}\right)$ plasmas, the wake has sufficiently low phase velocity to begin "dredging" electrons from the bulk population, resulting in a more chaotic and "black" wave. We thus adopt the designations of "blue" and "black" waves to qualitatively distinguish the physics of wakefield acceleration in the low- and high-density regimes, respectively. Between these two extremes additionally lies a transitional "grey" state.

To study the distinct physics of the high-density regime and its transition from the low-density regime, we employ the particle-in-cell (PIC) code EPOCH to model the injection of a laser down the axis of a bundle of CNTs. For simplicity the laser wavelength is taken to be 1 micron. (The corresponding laser frequency is then $1884 \mathrm{THz}$.) The specification of a bundle is necessary because while a reasonably achievable laser spot size is on the order of microns, the upper range of CNT diameters is typically tens of nanometers.

Establishing a firm conceptual foundation in this physics first requires understanding of the case where one spatial dimension (and three velocity dimensions) are employed. This work is also primarily interested in the phase-space structure of accelerated electrons, rather than the real-space evolution of the laser pulse and wakefield. While two spatial dimensions are used in Section 4, the main focus of this work requires only a 1D treatment. This model also allows a rough representation of carbon nanotubes in one spatial dimension. More complex phenomena that occur in 2D, such as strong self-focusing [30] and holeboring of ions [26], are not examined. Such 2D effects beget other effects, such as collapse of the laser pulse [31], which can lead to an expanding cloud of electrons. These effects and others [32], such as magnetic vortex physics [33] and prominent ion motion, are also more typical of the regime of ultra-intense pulses, which is not an emphasis of this work. Peak ion energy achieved in this study is typically $0.1 \mathrm{MeV}$. Comparisons to ion-acceleration schemes, such as TNSA and RPA [34,35] are not addressed here. Indeed, ions are essentially stationary in this study. 
For this simulation setup, the laser is injected from vacuum through an impedancematching boundary into a uniform plasma of electrons and protons with temperature $T=100 \mathrm{eV}$. Such a configuration represents a simple and idealized case that can be analyzed easily, yet contains the crucial physics. This scheme contrasts with some past efforts in which the laser was injected into a density ramp that peaked near the critical density [31]. In this case, the laser pulse collapsed before reaching the region of critical density owing to important $2 \mathrm{D}$ effects. Here a uniform density is used to examine the physics of laser-plasma interaction at the full critical density. Additionally, as opposed to initializing the laser inside the plasma, the vacuum injection scheme may better reflect the experimental reality for the high-density case near the critical density if we take as a target a porous nanomaterial $[36 ?, 37]$, for which the injected laser has $v_{g} \sim 0$. Care is taken that the essential laser-plasma physics is unchanged between the cases of vacuum injection and laser initialization inside the plasma.

The laser is taken to have a resonant pulse length with functional dependence $E_{y}=E_{0} \sin (k x-\omega t-\phi) h(x, t)$, where $h(x, t)$ is a resonant flat-top profile, and $\phi$ is an optional phase, which can be identified as the carrier envelope phase (CEP). By default $\phi$ is set to zero. (The oscillatory component of $E_{y}$ thus grows from zero as the laser enters the simulation domain.) A resonant pulse is half the length of the plasma wavelength $\lambda_{p}=2 \pi c / \omega_{p}$. The remaining laser and plasma quantities are then controlled through two parameters: the laser intensity $a_{0}$ and the critical density ratio $n_{\mathcal{c}} / n_{e}$. Position in the simulation domain is indicated by the value $x$. The laser is injected at $x=0$, and "forward" and "backward" correspond to the directions $+\hat{x}$ and $-\hat{x}$, respectively.

The total 1D domain size is 16 micron, representing an equal number of laser and plasma wavelengths (16) for $n_{e}=n_{c}$. Similarly, the plasma frequency $\omega_{p}$ is equal to the laser frequency. The domain is divided among 7187 cells to adequately resolve the Debye length. Each cell is initialized with 4000 electron and 40 ion pseudo-particles, the former being the physical interest and the latter being relevant for noise reduction.

The resonant pulse used in this case is motivated by a desire for simplicity and consistency with the approach typically used in the low-density regime. A resonant laser pulse in a plasma near the critical density $\left(n_{c} / n_{e} \leq 2\right)$ must necessarily be sub-cycle, or at most single cycle. While sub-cycle lasers have been demonstrated experimentally [38,39], such a setup would be generally difficult to implement, particularly in a fiber laser [16]. Another concern arising from a sub-cycle laser pulse is the influence of the initial laser phase $\phi$. Care has been taken to ensure that choice of $\phi$ does not affect the qualitative physics, even for $n_{c} / n_{e}=1$. One can show analytically that the initial ponderomotive kick felt by the electrons lies in the same direction as the laser Poynting vector, creating a tendency toward uniformity across various values of $\phi$.

In the "blue" wave case, where $n_{e} \ll n_{c}$, electrons that are trapped and accelerated form an ordered and repeating structure, with the highest-energy electrons reaching the theoretically expected momentum of $p_{x}^{\max } \approx m_{e} c \sqrt{\left(2 g\left(a_{0}\right) n_{c} / n_{e}\right)^{2}-1}$ [2]. Similarly, the longitudinal electric field forms a coherent wake structure, with the field saturating near the expected value [40]. The "black" wave case shows qualitatively different behavior and invites closer inspection. For a plasma at the critical density $n_{e}=n_{c}$, snapshots during the early (Figure 2a) and late (Figure 2b) stages are shown. The phase space density is plotted in units of pseudo-particles per $p_{x} / m c \times x / \lambda_{p}$. A run of this type was first made in prior work [18], and a similar run is produced here to examine the acceleration mechanism more closely. The group velocity of the laser is reduced to zero, $v_{g}=0$, and the dephasing the pump-depletion lengths are reduced to less than one plasma wavelength, $L_{p, d} \lesssim \lambda_{p}$. This latter effect restricts the laser-plasma interaction to within one plasma wavelength. Streams of low-energy $(\Delta \mathcal{E} \sim 100 \mathrm{keV}$ ) electrons ejected from the site of laserplasma interaction replace the long train of orderly trapped electrons seen in the "blue" wave case. Ejection of electrons occurs roughly every plasma period. The generation of low-energy electrons indicates strong coupling between the laser and the bulk population 
of electrons. The donation of a net bulk momentum to the electrons suggests the presence of an effective viscosity.

This behavior, applicable where $n_{c} / n_{e} \approx 1$, clearly signals a departure in qualitative physics from that of the regime of typical wakefield acceleration, $n_{c} / n_{e} \gg 1$, particularly where the laser enters the plasma medium. Rather than penetrating the plasma without significant hindrance as occurs in the low-density regime, in the high-density regime the laser has group velocity $v_{g} \ll c$ and plows the bulk electron population forwards, forming a large spike in electron density $\left(\delta n_{e} / n_{e} \approx 3.5\right)$. As in the low-density case, ions essentially remain in place. This density spike creates a longitudinal electric field of approximately twice the strength of that in the low-density case and causes the reflection of a substantial portion of the laser. This powerful initial kick to the plasma establishes a strong longitudinal sheath oscillation of electrons in the range $0 \leq x \lesssim \lambda_{p}$.

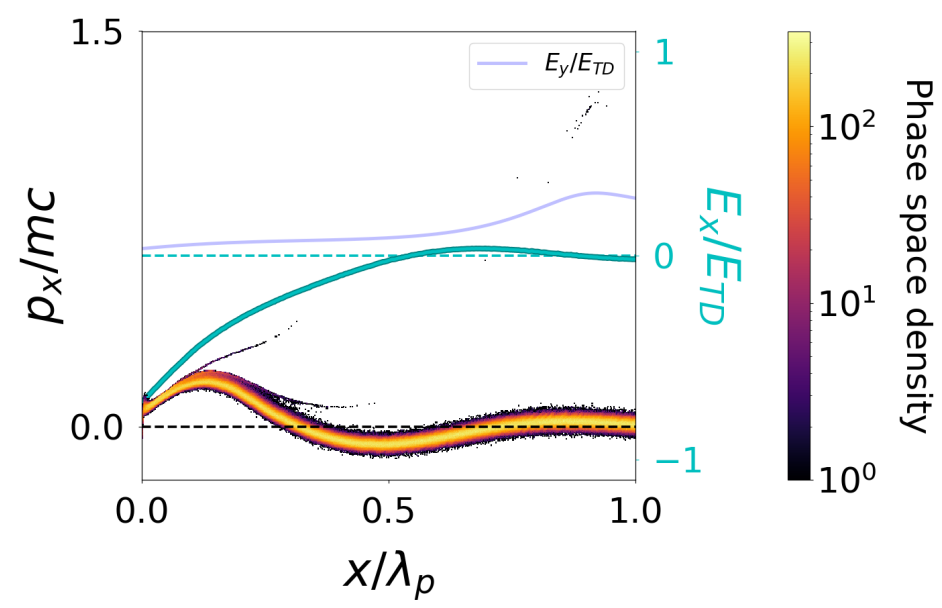

(a)

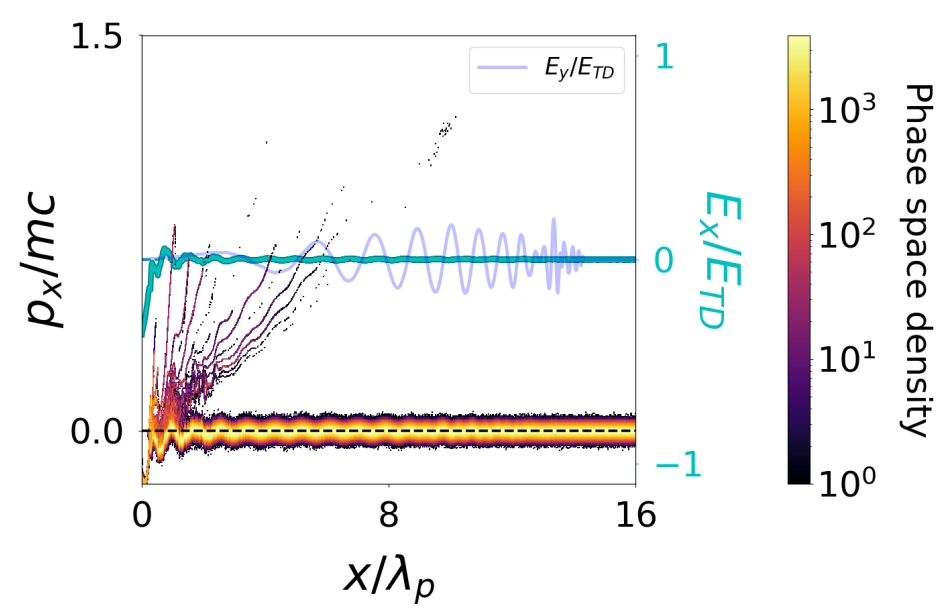

(b)

Figure 2. A snapshot of the electron phase space $p_{x}$ vs. $x$ and field structure of the high-density ("black") case $n_{c} / n_{e}=1$ of a $1 \mathrm{D}$ carbon nanotube with laser intensity $a_{0}=1$ at early (a) and later (b) stages. The heatmap shows warmer colors for higher phase-space density. The longitudinal $E_{x}$ (green) and laser $E_{y}$ (translucent blue) fields are plotted according to the right axis. The plasma wavelength is equal to $\lambda_{p}=1$ micron. (a) is zoomed to $0 \leq x \leq \lambda_{p}$ from (b). A similar run is made in previous work [18]. 
The formation of the sheath occurs when a strong restoring electric field then causes the density spike to rebound towards the left edge of the plasma (the entrance of the plasma domain). While this rebound occurs, a small population of electrons is accelerated to the energy $\Delta \mathcal{E}$ expected from traditional 1D wakefield theory. Simultaneously, many electrons are expelled from the left edge of the plasma, establishing a sheath [17] at the plasma edge. The intensity of this sheath is comparable to that of the initial laser amplitude. This sheath then continues to oscillate longitudinally, with each oscillation accelerating a stream of electrons to low energy $(<100 \mathrm{keV})$. These electrons are then ejected from the site of oscillation in the forward (right) direction. The presence of the sheath ensures that these electron streams travel nearly exclusively in the forward direction. (If the sheath is not located at the plasma edge, a significant number travel backwards.) Figure $2 a$, a snapshot of the electron phase space and fields zoomed to the range $0 \leq x \leq \lambda_{p}$, shows the beginning of this process, where the first streams of accelerated electrons are visible. As the oscillation continues, more streams accumulate, building up the phase-space distribution in Figure $2 b$, which shows the full simulation domain. In the later stages, the electron acceleration becomes increasingly turbulent until the oscillation is finally exhausted after about 30 plasma periods. The full evolution of the sheath and electron density is shown in Figure 3. While the individual energy of the accelerated electrons is low compared to that of the low-density case, the total energy imparted to the accelerated electrons can potentially be higher. This total imparted energy represents about $12 \%$ of the total injected laser energy, though it should be kept in mind that an such efficiency value is inherently a rough estimate in a 1D simulation. This understanding of the electron dynamics associated with sheath formation should also be useful for the understanding of related ion acceleration dynamics $[1,6]$. The sheath acceleration mechanism observed here is also reminiscent of that in cases examined previously [26].

The transition to sheath acceleration from typical wakefield acceleration represents a sharp division of qualitative regimes of wakefield physics. Pursuing this point further, we can attempt to find a quantitative means of discriminating these regimes. Comparing Figure 2 with that of the ordered structure of typical, low-density wakefield acceleration suggests a quantitative index related to the entropy of the phase-space structure; the electron phase space in the low-density case is highly ordered compared with that of the high-density case. In general however, comparing the entropy of two distributions also requires an accounting for the mean kinetic energy of the distributions. Thus, as an index for discriminating the "blue" and "black" regimes, we propose a "darkness" metric $D$. This quantity $D$ is defined as the specific momentum entropy $D=S /\langle K\rangle$, where $S=-\int_{-\infty}^{\infty} \ln \left[f\left(P_{x}\right)\right] f\left(P_{x}\right) d P_{x}$ is the differential Boltzmann entropy of the longitudinal momentum distribution $f\left(P_{x}\right)$, where $P_{x}=p_{x} / p_{T}$ is the longitudinal momentum normalized to the thermal momentum $p_{T}=\sqrt{m_{e} T_{e}}$, and $\langle K\rangle=\int_{-\infty}^{\infty}\left[\sqrt{1+P_{x}^{2} /\left(m_{e} c / p_{T}\right)^{2}}-1\right] f\left(P_{x}\right) d P_{x}$ is the average electron kinetic energy per particle normalized to $m_{e} c^{2}$ considering only the contribution from $p_{x}$. The distribution $f\left(P_{x}\right)$ is normalized according to $\int_{-\infty}^{\infty} f\left(P_{x}\right) d P_{x}=1$. This index $D$ does not take into account the dependence of the total laser energy content on the plasma wavelength $\lambda_{p}$; as $\lambda_{p}$ increases for lower densities, the laser pulse length increases to maintain a resonant length. Instead, by keeping the laser always to a resonant pulse length, the plasma excitation mechanism is held constant.

For a scan over the density values $n_{c} / n_{e} \in[0.5,14]$ at $a_{0}=1$, Figure 4 shows the approximately final values for the index $D$ normalized to the initial value $D_{0}$, which is the same for each data point and has an analytic form for a Maxwellian distribution. One point in the overdense regime $\left(n_{c} / n_{e}=0.5\right)$ has been added as well for cautious comparison of the "black" and super-critical regimes. As $n_{c} / n_{e} \rightarrow \infty, s / s_{0} \rightarrow 0$, while as $n_{c} / n_{e} \rightarrow 0$, $D / D_{0}$ climbs to a large value. These limits reflect the substantive difference in each regime. For "blue" waves, the average electron energy grows much faster than the momentum entropy, owing to the development of a typical wakefield phase-space structure. For "black" waves in contrast, disorder in phase space dominates growth in average kinetic energy and becomes ever more severe for increasing plasma density. The "darkness" index $D / D_{0}$ 
for "blue" waves thus asymototes to zero while that for "black" waves is characterized by finite size tending to a large value. Between these two extremes, a "grey" wave state can exist, which shows both aspects of bulk flow and traditional wakefield acceleration.

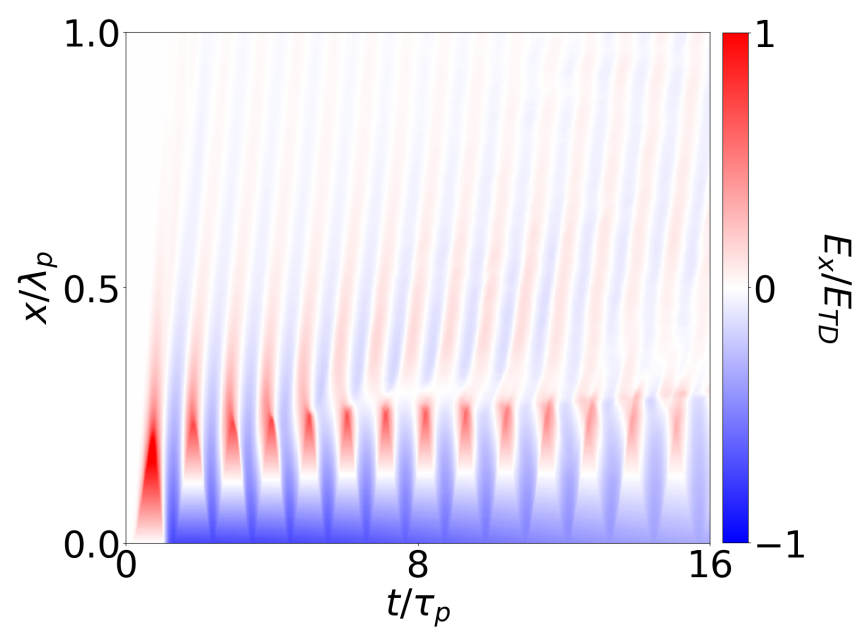

(a)

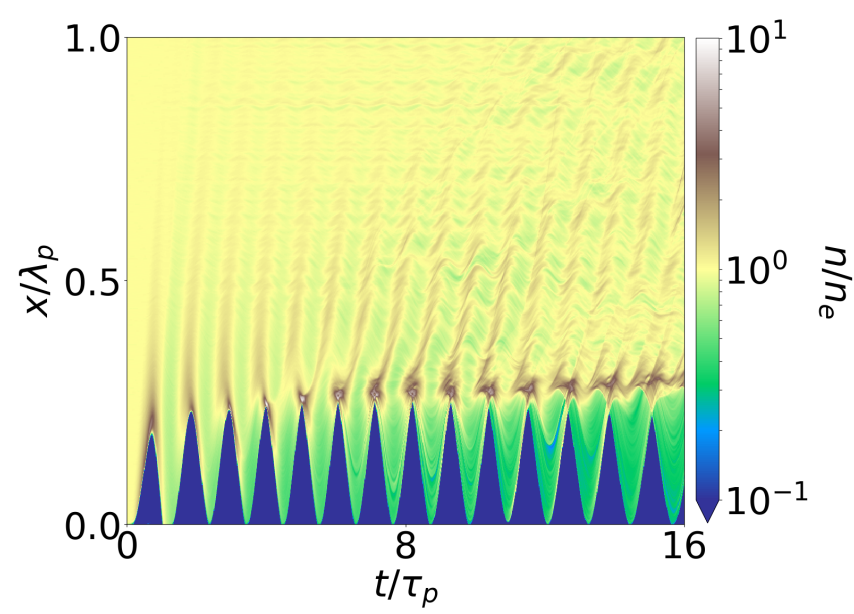

(b)

Figure 3. The evolution in space and time of the longitudinal electric field $E_{x}$ (a) and electron density $n(\mathbf{b})$. The electric field is normalized with respect to the Tajima-Dawson field $E_{T D}$, and the electron density $n$ is normalized to the initial uniform density $n_{e}$. The downward arrow on the color bar of $(\mathbf{b})$ reflects that $n$ extends to zero in the deep blue areas. The time domain is normalized with respect to the period of plasma oscillation, $\tau_{p}=2 \pi / \omega_{p}$.

This index may then provide a guide to the most appropriate regime in which to operate for a particular application. For the generation of a mono-energetic, high-energy electron beam, $D / D_{0} \ll 1$ is desirable, as a "blue" wave will cleanly accelerate a small population of electrons to extremely high energy. Being mindful of the dephasing length, one can then ensure that the accelerated electrons are captured at peak energy. In contrast, for some medical applications, such as in a cancer-treatment scheme where a source of radiation is brought directly to the cite of a tumor, a significant dose of low-energy (shallow-penetrating) electrons is desirable, with less constraint on the beam quality and electron energy distribution. One is attracted in this case to the "black" regime, which has $D / D_{0} \sim 1$. For long, low-intensity laser pulses, as would be amenable to a fiber laser, this regime can also be accessed even at moderate plasma densities $\left(n_{c} / n_{e} \approx 10\right)$ due to Raman forward scattering [18]. 


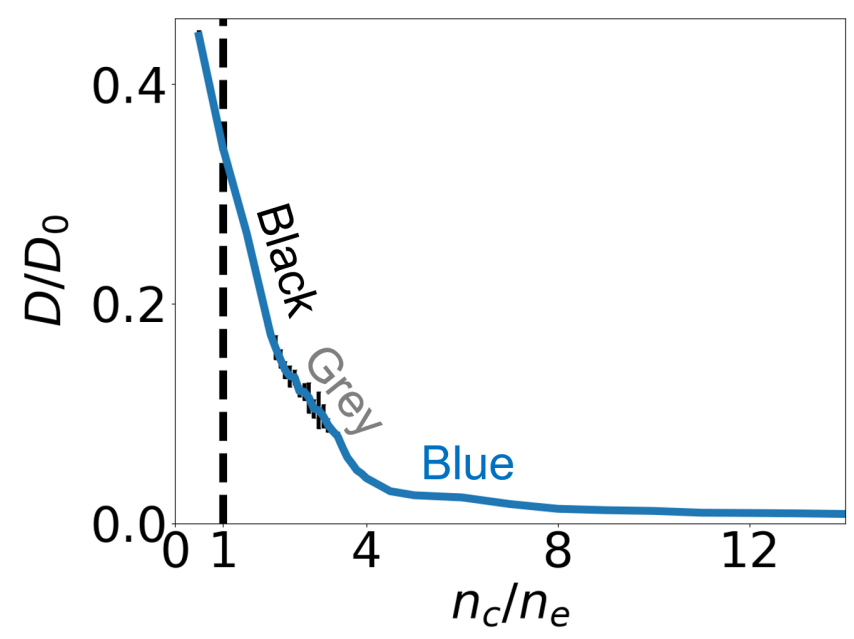

Figure 4. The specific entropy ("darkness") index $D$ normalized to its initial value $D_{0}$ for a scan of the density ratio values $n_{c} / n_{e} \in[0.5,14]$ for laser intensity $a_{0}=1$ and a resonant pulse length. The plotted index is the mean of the calculated index for the last ten time steps in each run. The black error bars represent one standard deviation of this set of averaged values. Rough indications of the wave type for particular regions are given in text. Because the plasma is initialized with the same temperature in each case, the initial index $D_{0}$ is the same for every data point. The critical density is marked by a dashed line.

\section{Laser Intensity Scaling}

Apart from plasma density, the second chief parameter determining the nature of the wakefield response is the laser intensity $a_{0}$. To understand wakefield physics at high density, it is thus also necessary to understand the scaling of accelerated electron energy $\Delta \mathcal{E}$ with respect to $a_{0}$. For medical applications, it is also necessary to understand the electron energies available for various laser intensities, particularly $a_{0}<1$. Two cases are considered: the low-density "blue" case of $n_{c} / n_{e}=10$ and the moderate-density "grey" case of $n_{c} / n_{e}=3$. For each case $a_{0}$ is scanned logarithmically over the range $a_{0} \in[0.1,10]$ and the maximum electron energy is recorded.

The expected functional dependence of $\Delta \mathcal{E}\left(a_{0}\right)$ has thus far been represented simply by $g\left(a_{0}\right)$. Here we compare the case $g\left(a_{0}\right)=\sqrt{1+a_{0}^{2}}-1$ to the results of the scan over $a_{0}$. Of particular interest for comparison with the simulation results is that this function has two slope regimes: $\ln g / \ln a_{0}=2$ for $a_{0} \ll 1$ and $\ln g / \ln a_{0}=1$ for $a_{0} \gg 1$.

The results of the scan are shown in Figure 5. First for the low-density ("blue") case (Figure 5a), the transition in slope is indeed seen, and the simulation results are in general agreement with $g\left(a_{0}\right)$. The moderate-density ("grey") case (Figure $5 \mathrm{~b}$ ) also shows rough agreement with $g\left(a_{0}\right)$. Notably, for the low-density case, a sharp transition in maximum electron energy is seen around $a_{0}=1$. This transition may be indicative of the "switching on" of electron trapping that occurs once the laser amplitude enters the relativistic regime at $a_{0}=1$; for $a_{0} \ll 1$, substantial electron trapping does not occur for typical LWFA. In contrast, at higher density (Figure $5 b$ ), the transition is less prominent, and electron energy for $a_{0} \ll 1$ is higher than that given by $g\left(a_{0}\right)$. Because the electron acceleration mechanism in this regime has shifted more to the sheath acceleration typified in Figure 2b, which does not have an intensity-based "switching on" transition, but can instead accelerate electrons even at very low intensities owing to the slow laser group velocity $v_{g} \lesssim v_{T}$, a less abrupt transition around $a_{0}=1$ in this case is expected. As an additional consequence, the sheath acceleration is able to accelerate electrons with comparative efficiency in the regime $a_{0} \ll 1$, leading to the apparent acceleration enhancement above $g\left(a_{0}\right)$ in Figure $5 \mathrm{~b}$. In the regime of very large $a_{0}$, it should also be noted that other work [41] has found that the scaling of electron energy should follow $a_{0}^{2}$ from ulta-relativistic effects. 


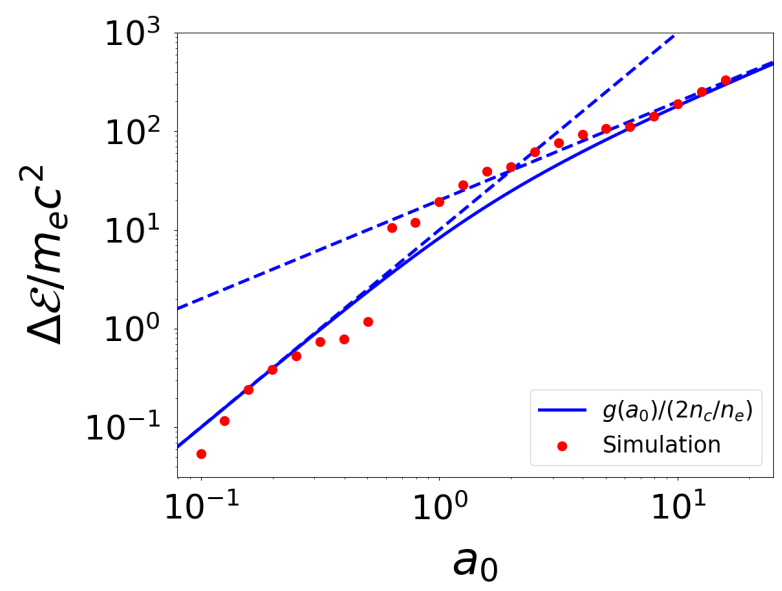

(a) $E_{\max }$ vs. $a_{0}$ for fixed ratio $n_{c} / n_{e}=10$.

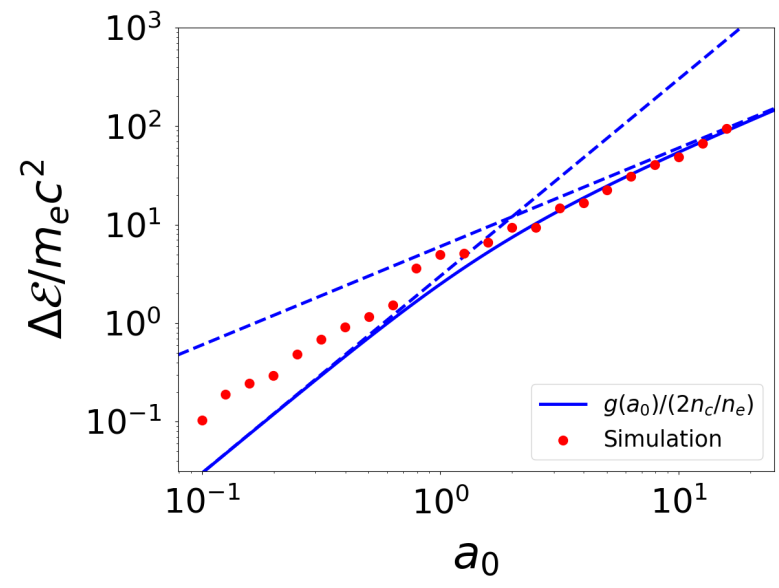

(b) $E_{\max }$ vs. $a_{0}$ for fixed ratio $n_{c} / n_{e}=3$.

Figure 5. The maximum electron energy as a function of laser intensity $a_{0}$ for two density cases: $n_{c} / n_{e}=10(\mathbf{a})$ and $n_{c} / n_{e}=3(\mathbf{b})$. The maximum energies (red dots) are compared with the function $g\left(a_{0}\right)$ (blue solid line). The blue dashed lines represent the asymptotic behavior of $g\left(a_{0}\right)$ for $a_{0} \ll 1$ and $a_{0} \gg 1$.

These results suggest that an $a_{0}$ between 0.1 and 0.8 gives the best results for achieving electrons with $\Delta \mathcal{E}<1 \mathrm{MeV}$ while still allowing the density ratio to be varied, such as would be desired for medical applications featuring shallow beam penetration.

\section{High Density with Perpendicular Carbon Nanotubes}

Thus far we have examined the mechanics of wakefield acceleration in the highdensity regime by modeling laser injection parallel to a bundle of CNTs. We now consider an alternative arrangement where the CNTs are oriented in the direction perpendicular to that of the laser propagation. To examine this case, we expand our simulation scheme to include two spatial dimensions. The properties of CNTs, particularly an effective onedimensional conductivity, allow for the straightforward modeling of a high-density target of perpendicularly oriented CNTs in the arrangement of Figure $1 \mathrm{~b}$ in a PIC simulation. This arrangement is investigated using an implementation of the PIC code EPOCH with two spatial dimensions. An array of CNTs is modeled as periodic discrete bars of plasma with specified width and height in the $x$ and $y$ directions, respectively, as is shown schematically in Figure 6. The 2D domain size is 8 micron ( 4000 cells) in the $x$ direction and 12 micron (6000 cells) in the $y$ direction. The spatial resolution is thus $2 \mathrm{~nm}$ in each direction. The aperture size is 4 micron. A total of approximately one million pseudo-particles are used, 
each representing $10^{12}$ real particles. The pseudo-particles are initially concentrated inside the nanotubes, with no particles initialized elsewhere.

Modifications to the code are made to enforce the one-dimensional conductivity of the CNTs by allowing electron motion and momentum change only in the $y$ direction while an electron is inside a CNT. In order to emulate electron field emission, the boundary corresponding to the tip of each CNT is left open to allow electrons to spill out and leave the CNT as shown in Figure 6. This approximation is justified by the high laser fields of order $10^{10} \mathrm{~V} / \mathrm{m}$ or greater and the low work function of the CNT. These boundaries and restrictions on movement allow emission from the CNT tip only when the laser is polarized parallel to the CNT axis, a phenomenon found in previous experimental results [42]. The opposite boundary, where the CNTs touch the edge of the simulation domain in the $y$ direction, is made periodic so that the upper and lower CNTs can exchange electrons. This boundary condition models the high parallel conductivity of the CNTs and mitigates space-charge effects that might result from the finite length of the CNTs used in the simulation. The CNTs are separated with equal spacing. The surplus space provided by this arrangement allows flexibility in the CNT spacing and consequently the effective electron density perceived by the laser while holding the total number of CNTs constant. The perceived electron density is calculated as the average electron density taking into account the CNTs and the vacuum gaps between them. The density of the CNTs themselves is taken to be $10^{22} \mathrm{~cm}^{-3}$, and the CNT width is $10 \mathrm{~nm}$.

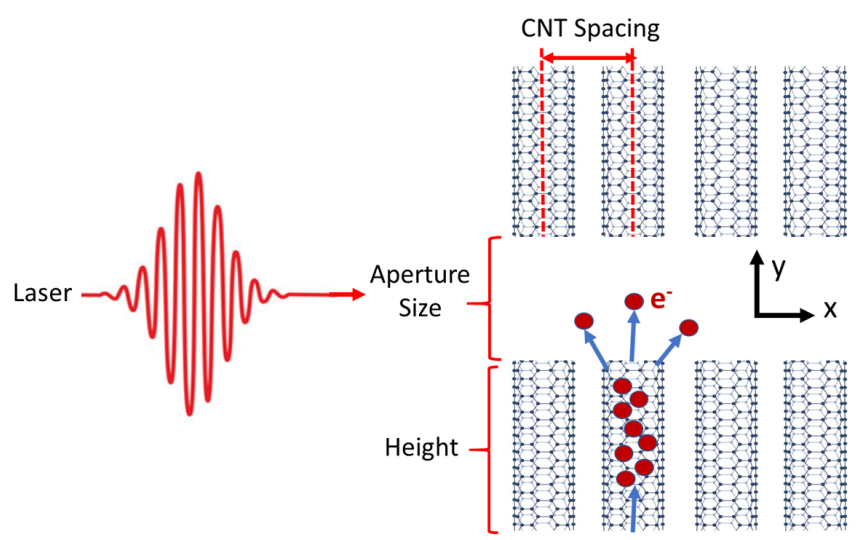

Figure 6. Visual diagram of the CNT-laser setup where a laser is injected perpendicularly to an array of CNTs.

As we wish to explore super-critical densities with this arrangement of CNTs, here we switch to the characterizing ratio $n_{e} / n_{c}$, where $n_{e}$ is the average perceived electron density accounting for the CNTs and the empty space between them. Figure 7 shows the change in this ratio with respect to CNT separation. The average density becomes equal to the laser critical density (and the ratio becomes unity) for a spacing of approximately $200 \mathrm{~nm}$. By reducing the spacing, the perceived electron density can easily exceed the laser critical density, reaching up to $n_{e} / n_{c}=10$ for the values surveyed.

To extract electrons from the CNTs, two lasers pulses (with a wavelength of 1 micron as before) are injected sequentially. The second laser pulse enters the simulation after the first pulse has fully exited (around 45 fs of total simulation time). Each has a Gaussian profile in time and width corresponding to a pulse length of $15 \mathrm{fs}$ and a spot size of 10 micron, respectively. Each pulse has $a_{0}=0.5$. The first pulse extracts electrons to form an electron cloud between the rows of CNTs. The second pulse then accelerates the electrons in the cloud. We scan over various parameters of the system in order to obtain energy scaling laws. Figure 8 shows the locations of electrons overlaying the laser electric field for one simulation at different times to visualize the effects of two laser pulses. The number of extracted electron pseudo-particles in the space between the CNTs in Figure 8b suggests an estimated extracted electron density of $10^{19} \mathrm{~cm}^{-3}$. 


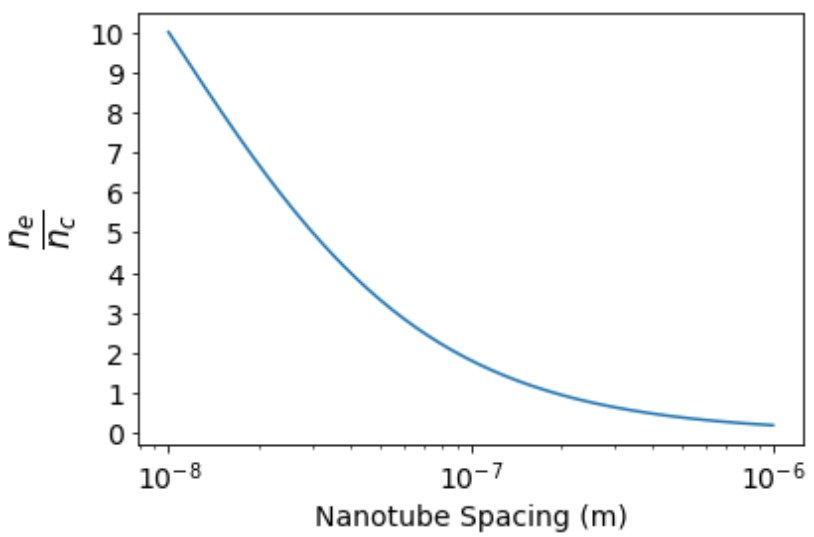

Figure 7. Ratio of perceived electron density to critical density $n_{e} / n_{c}$ as a function of the vacuum spacing between CNTs. (Note that this ratio is the reciprocal of that quoted in previous sections.) The density inside a CNT is $10^{22} \mathrm{~cm}^{-3}$, and the width of a CNT is $10 \mathrm{~nm}$. The laser wavelength is 1 micron.

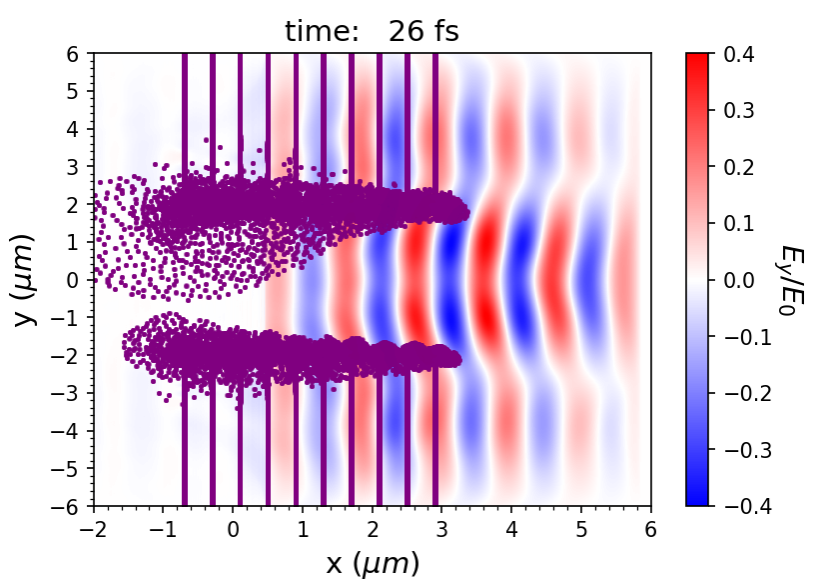

(a)

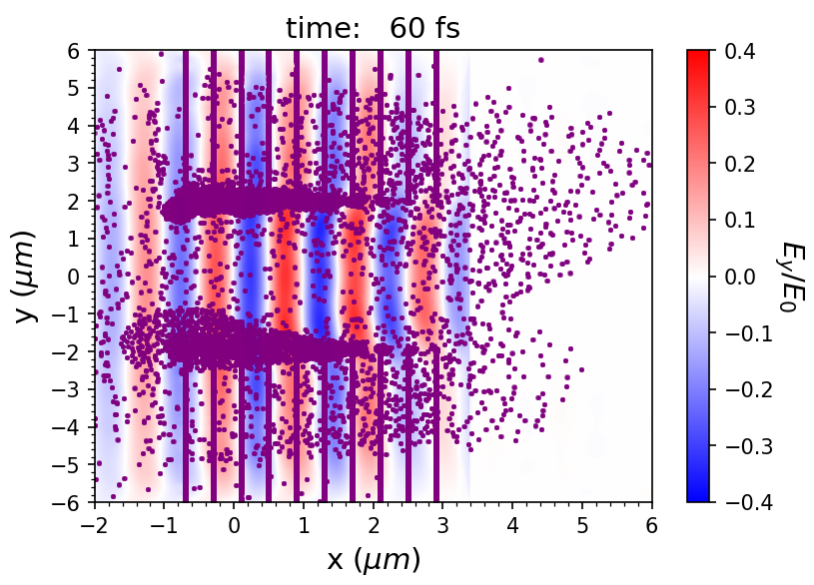

(b)

Figure 8. Example of simulation results with first laser extracting electrons from the CNTs (a) and a second pulse accelerating the extracted electrons $(\mathbf{b})$. The purple dots indicate the locations of individual electrons, and the colormap indicates the strength of the laser field. The laser field is normalized to the nominal peak amplitude $E_{0} \approx 1.6 \times 10^{12} \mathrm{~V} / \mathrm{m}$. 
Although the first laser pulse is primarily intended to extract electrons from the CNTs, it also has some ability to accelerate electrons, as is shown by the energy distribution in Figure $9 \mathrm{a}$, which corresponds to the moment that the first laser pulse has left the simulation and the second pulse has not yet entered. The momenta of these accelerated electrons lies mostly in the $\pm \hat{y}$ direction. As the second laser reaches midway through the domain, it couples with the freed electrons and ponderomotively accelerates them in the $+\hat{x}$ direction. This second stage of acceleration produces a bump in the energy distribution at around $20 \mathrm{keV}$ and pushes the maximum energy to a higher value, as is shown in Figure $9 \mathrm{~b}$.

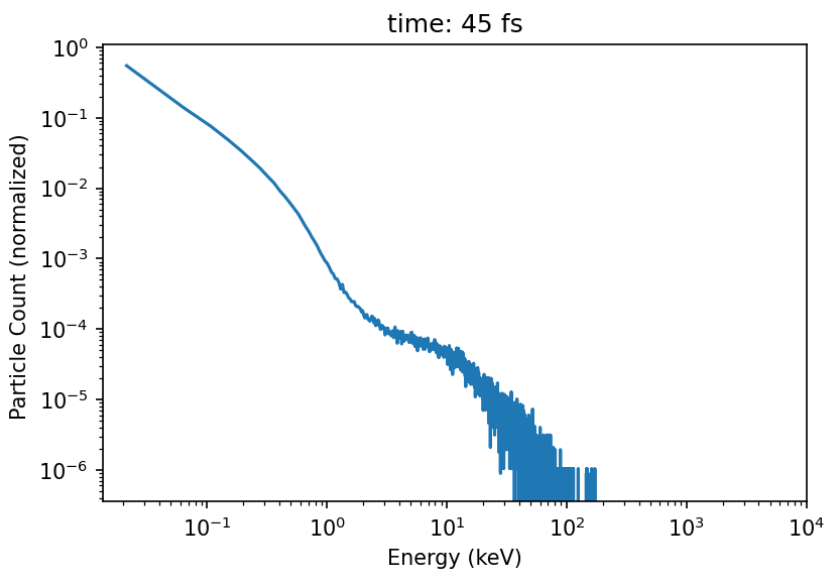

(a)

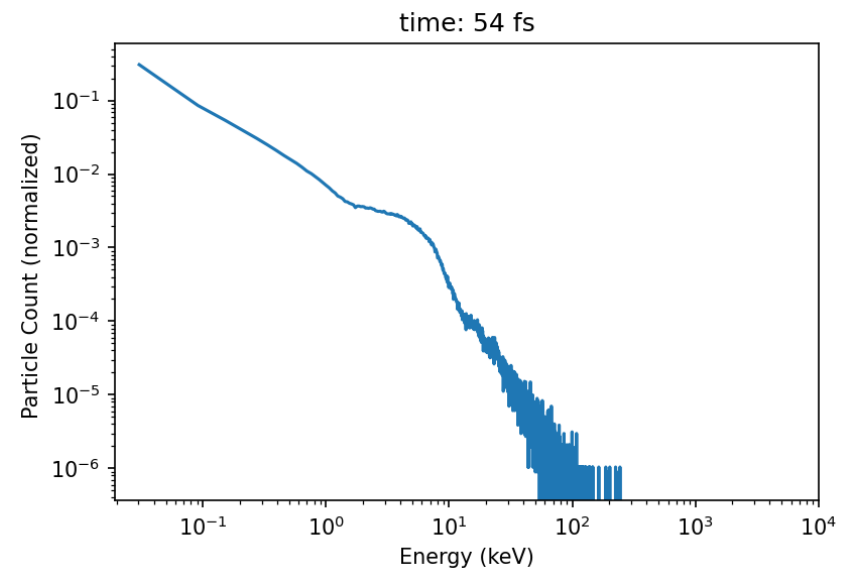

(b)

Figure 9. Example electron energy distributions at two times: after the first laser leaves simulation domain (a) and when the second laser reaches half way through the simulation domain (b).

The acceleration that occurs in the second stage requires some further remarks. Ponderomotive acceleration, as conceived in typical wakefield physics, is the result of a nonuniform and oscillating electromagnetic field and involves averaging of the accelerating field. In this case, however, acceleration in the $+\hat{x}$ direction can occur in a uniform laser field. This acceleration occurs via the instantaneous ponderomotive force given by $p_{y} B_{z}$, where $p_{y}$ for an electron is provided by the first laser pulse. In this manner, electrons with a favorable phase with respect to the laser can receive a kick in the $+\hat{x}$ direction. In a vacuum, such electrons cannot remain in phase with the laser (as is possible in typical wakefield acceleration) but nonetheless retain a positive $p_{x}$.

Another consideration affecting electron acceleration is the perceived CNT electron density. Figure 10 shows the number and maximum energy of accelerated electrons with 
respect to the perceived ratio $n_{e} / n_{c}$. The ratio is varied by changing the CNT spacing. For $n_{e} / n_{c}<1$, the laser is able to penetrate deep into the the CNT structure and extracts electrons in proportion to the total number of CNTs, as is shown in Figure 10a. When the perceived electron density exceeds the critical density, laser propagation through the CNT structure is impeded, and consequently fewer electrons are extracted. Note that electron recapture by the CNT boundaries causes a decrease in extracted electrons between roughly 20 and $60 \mathrm{fs}$.

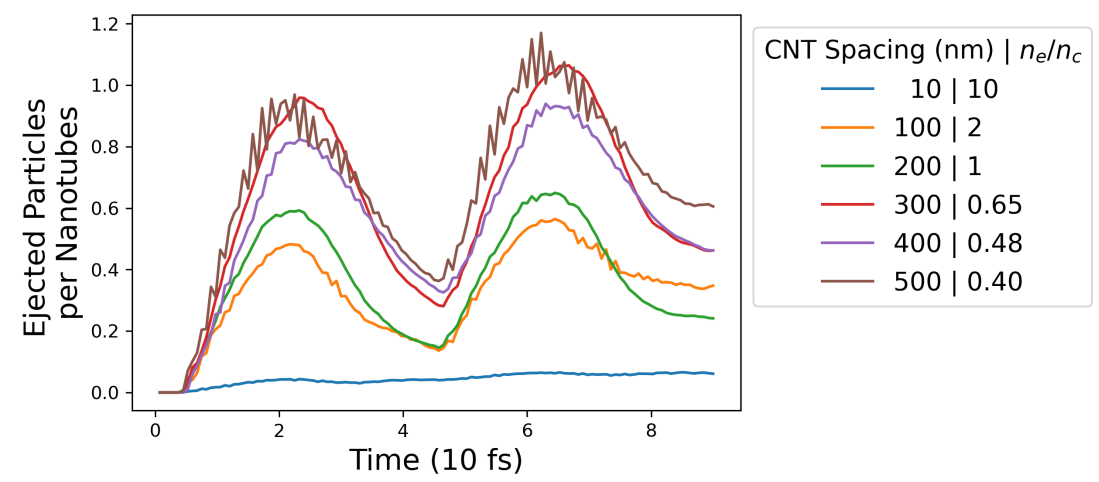

(a)

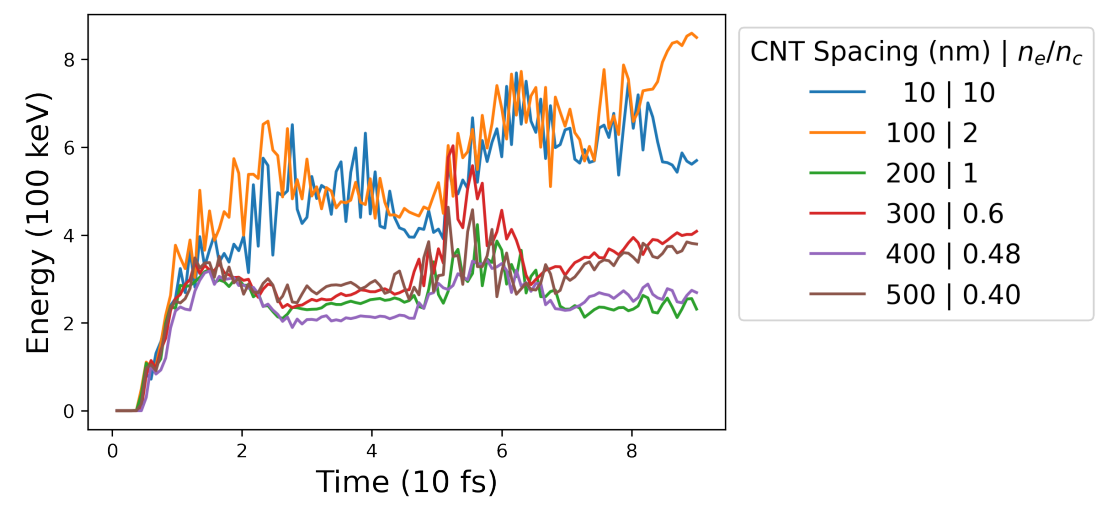

(b)

Figure 10. Number of extracted electrons (a) normalized to total number of CNTs in the simulation as a function of time and maximum energy of electrons (b) over simulation time for various values of CNT spacing. The first laser reaches CNTs in the first few femtoseconds. The second laser reaches the CNTs at around $45 \mathrm{fs}$. The laser intensity is $a_{0}=0.5$.

For the same simulations, maximum electron energy is shown in Figure 10b. Two regimes are evident; a significant transition in the maximum electron energy occurs for $n_{e} / n_{c}>1$. In light of this observation and the preceding arguments, we propose an energy scaling law to roughly predict the energy gain $\Delta \mathcal{E}$ based on instantaneous ponderomotive acceleration as follows:

$$
\Delta \mathcal{E}=v_{e x} \Delta p=a_{0}^{2} m c^{2}\left(\frac{v_{e x}}{c}\right) \omega \Delta t,
$$

where $v_{e x}$ is defined as the maximum velocity in the $+\hat{x}$ direction of all particles in the system, $\Delta \tau=15 \mathrm{fs}$, and $\omega=2 \pi c / \lambda \approx 1.8 \times 10^{15} \mathrm{~s}^{-1}$. The interaction interval $\Delta t$ is defined as

$$
\Delta t=\frac{c \Delta \tau}{c-v_{e x}}=\frac{\Delta \tau}{1-v_{e x} / c}
$$


Using Equation (2) in Equation (1), we find that:

$$
\Delta \mathcal{E}=\frac{1}{8} a_{0}^{2} \cdot(0.5 \mathrm{MeV}) \cdot\left(\frac{v_{e x}}{c}\right) \frac{\omega \Delta \tau}{1-v_{e x} / c}
$$

This scaling law compares well with simulation results as shown in Figure 11 for two different $n_{e} / n_{c}$ density ratios on the extreme ends (0.48 and 10$)$.

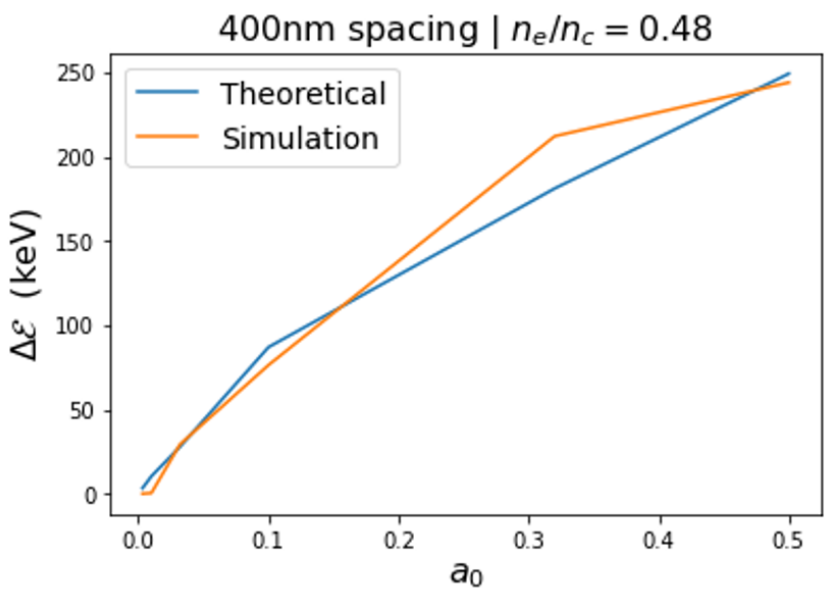

(a)

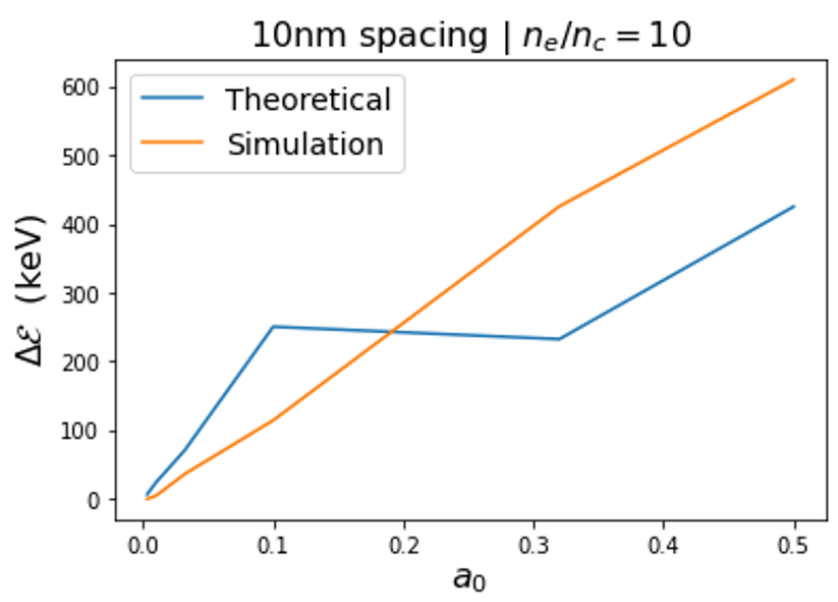

(b)

Figure 11. Comparison of maximum electron energies $\Delta \mathcal{E}$ with respect to $a_{0}$ from simulations to the theoretical scaling law prediction given in (3) for CNT spacing of $400 \mathrm{~nm}$ which correlates to $n_{e} / n_{c}=0.48$ (a) and for $10 \mathrm{~nm}$ spacing corresponding to $n_{e} / n_{c}=10.0(\mathbf{b})$.

An interesting idea to consider is that of resonance between the spacing and plasma wavelength of the CNTs. For the present CNT density of $10^{22} \mathrm{~cm}^{-3}$, the corresponding plasma wavelength is $\lambda_{p} \approx 334 \mathrm{~nm}$. This case is roughly represented by the red traces in Figure 10. The monotonic decrease in number of extracted electrons with CNT spacing in Figure 10a is interrupted for a spacing of $300 \mathrm{~nm}$. A similar phenomenon is seen in Figure 10b. While the super-critical traces appear to follow different qualitative behavior, the case of a spacing of $300 \mathrm{~nm}$ gives the greatest electron energy among the critical and sub-critical traces. These observations suggest a possible resonance between the CNT plasma wavelength and spacing. 


\section{Conclusions}

The majority of efforts involving plasma wakefield acceleration have focused on producing ever higher-energy electron beams, particularly for applications in particle accelerators. Notable experiments in this area include BELLA, FACET, and AWAKE (mentioned in such as $[40,43]$ ), which aim to reach $\mathrm{TeV}$ energies. To reach such high acceleration gradients, experiments typically use low-density plasmas $\left(n_{c} / n_{e} \gg 1\right)$ and $a_{0}>1$.

The opposite regime, that of LWFA near the critical density, remains comparatively unexplored. Nonetheless, this regime holds potential for innovative approaches in fields such as cancer therapy, in the form of LWFA-powered endoscopic electron therapy (or intraoperative radiation therapy). This regime also opens possibilities for highly localized, precise treatment of targets on the skin, such as for oncology or cosmetics, making use of extremely shallow-penetrating electrons. This latter case may be particularly tractable given the low electron energies required. Crucially in all of these applications, the source of radiation is brought close to the irradiation targets such as tissues to be treated, removing collateral damage to other tissues. Consequently the radiation need only have limited penetrative power. With electrons used as the source of radiation, the desired energies are then $\lesssim 1 \mathrm{MeV}$, which yields a penetration depth between microns and millimeters [18]. By tuning the laser intensity and plasma density, specific depths can be produced as desired. Here we have shown though a preliminary study that LWFA can indeed produce such electrons.

To do so, the plasma density must be close to the critical density $\left(n_{c} \sim n_{e}\right)$, a regime that can be accessed via a solid-state target such as a bundle of carbon nanotubes. In this regime the group velocity of a laser pulse becomes increasingly slow, and the laser-plasma interaction range reduces nearly to a single wakefield oscillation. As a consequence of these properties, the wakefield, rather than skimming a small number of electrons from the bulk distribution and accelerating them to high energy, instead dredges somewhat more deeply from the bulk, creating an effective viscosity and momentum transport. This situation manifests as a "black tsunami" in analogy to beach wave physics and represents a qualitative departure from typical wakefield physics. Upon slowing down near the shore, the wave begins to break, creates turbulence, and dredges the sea floor, creating a visibly black wave. Wakefield physics in the limit of $n_{c} / n_{e}=1$ manifests in a similar way, resulting in a churning wave of relatively low-energy electrons which can then be harnessed as a beam.

The gradual transition between the "black" and "blue" wakefield regimes, corresponding respectively to high and low plasma density, has been shown, along with the linear scaling of peak electron energy. The specific entropy metric $D$ has been proposed as a quantitative index for the regime of wakefield physics under consideration. In the low-density limit ("blue" waves), $D \rightarrow 0$, while near the critical density ("black" waves), $D$ becomes finite, possibly tending to a large value. Furthermore, a scan of maximum electron energy $\Delta \mathcal{E}$ over a range of intensities $a_{0}$ reveals a general agreement with the function $g\left(a_{0}\right)=\sqrt{1+a_{0}^{2}}-1$, derived from the ponderomotive potential, despite additional complexities and the limitations of a 1D simulation geometry. The difference in the mechanism of acceleration between the high- and low-density regimes is also manifested in the maximum electron energies attained in the two cases in Figure 5.

The recent development of coherent networks of fiber lasers (CAN) [16] has allowed LWFA research to branch into new fields of medical applications. However, two chief limitations must be addressed for a medical fiber laser system: laser intensity and pulse length. Fiber lasers have stringent intensity limitations, with a maximum allowed individual fiber intensity likely less than $10^{14} \mathrm{~W} \mathrm{~cm}$. This limitation can be mitigated through the use of many coherently added fibers and by retreating from the critical density to a more modest plasma density (such as $n_{c} / n_{e}=10$ ). Even with these factors, the ultimate intensity would likely remain in the regime of $a_{0}<1$. Limitations on pulse length are also stringent; the shortest pulse length likely achievable in a fiber laser system is around $100 \mathrm{fs}$, which is 
several times longer than was used in Figure 2. Fortunately, Raman scattering effects and self-modulation may allow the "black", low-energy electron regime to be accessible for a long pulse, even at very low intensity $\left(a_{0} \ll 1\right)$ and low density $\left(n_{c} / n_{e}=10\right)[18,44]$.

The bulk of this work addressed a scheme were a laser is injected parallel to the axis of a modeled bundle of carbon nanotubes. With these limitations of fiber lasers in mind, however, exploratory simulations were also made to investigate an alternative, 2D arrangement of carbon nanotubes (CNTs), also allowing attainment of densities much higher than those possible in gas plasmas. CNTs were selected for various advantageous electrical properties, such as an effective one-dimensional conductivity. In this arrangement, two laser pulses travel through a gap between two sequences of CNTs with notably the laser electric field directed parallel to the CNT axis. The first laser pulse extracts electrons from the CNTs, and the second pulse accelerates the electrons by an instantaneous ponderomotive mechanism that is atypical of wakefield physics. Scaling for the number of electrons emitted and maximum electron energy with respect to the perceived electron density was investigated, leading to a proposed energy scaling law. These results suggest that such an arrangement of CNTs may be able to efficiently generate a large flux of low-energy electrons as would be useful in a medical application. Additionally, the high critical density of the laser and the high density of the CNTs compared to the background air density suggest that a device based on this scheme may not need to impose an internal vacuum, which is much more amenable to medical applications. We invite further investigation of using CNTs in this arrangement for generating large fluxes of low-energy electrons.

Other challenges remain. This work has addressed only the most fundamental aspects of LWFA near the critical density. The population of accelerated electrons generated by LWFA at high density is non-monoenergic and probably of high emittance. Additionally, treatment of higher-dimensional effects such as focusing and hole-boring will be necessary for any ultimate medical application, and thus must therefore await future work. However, we emphasize that the remarkable trend in specific entropy as the plasma density approaches the critical density may not have been noticed with the inclusion of higherdimensional effects in a mixed effort. In this sense, our study has followed in the spirit of Boltzmann [45] in his development of Boltzmann entropy. We may also strive to further increase energy efficiency. Toward such a purpose we may wish to employ a graded density of plasma to control the phase gradation of the wakefield [46,47]. Nonetheless, interesting physics has already emerged from these efforts, and the richness of a new regime is evident.

Author Contributions: Conceptualization, T.T.; Formal analysis, B.S.N. and E.B.-V.; Funding acquisition, T.T.; Investigation, B.S.N., E.B.-V., S.H., K.C., G.D., K.G., D.H., G.H., G.L., D.L., P.M., E.N., G.P., M.S., J.S., J.T. and K.T.; Methodology, T.T.; Project administration, T.T.; Resources, T.T.; Validation, T.T.; Writing-original draft, B.S.N., E.B.-V., S.H. and T.T.; Writing-review \& editing, B.S.N. All authors have read and agreed to the published version of the manuscript.

Funding: This work was partially supported by the Norman Rostoker Fund at UCI.

Institutional Review Board Statement: Not applicable.

Informed Consent Statement: Not applicable.

Data Availability Statement: Not applicable (this study does not report any available data).

Acknowledgments: The present paper arose from the term-project efforts of the students in the tri-campus (UCI, UCLA, UCSD) graduate physics course Special Topics in Plasma Physics PHY249, "Nonlinear Plasma Physics" (Winter, 2019), led by the instructor T. Tajima. We also tried to tie plasma physics with other disciplines such as medical physics and geophysics to broaden the students' experience in physics. The tri-campus plasma physics graduate course was launched in academic year 2018, and this course was one of three such courses. The materials are partially available on a Google Drive upon request. We are thankful for discussions with G. Mourou, D. Strickland, J. Wheeler, J.C. Chanteloup, D. Roa, X. Yan, N. Beier, and A. Nečas. 
Conflicts of Interest: The authors declare no conflict of interest. The funders had no role in the design of the study; in the collection, analyses, or interpretation of data; in the writing of the manuscript, or in the decision to publish the results.

\section{References}

1. Tajima, T.; Nakajima, K.; Mourou, G. Laser Acceleration. La Riv. del Nuovo Cimento 2017, 40, 33-133. [CrossRef]

2. Tajima, T.; Dawson, J.M. Laser Electron Accelerator. Phys. Rev. Lett. 1979, 43, 267-270. [CrossRef]

3. Strickland, D.; Mourou, G. Compression of amplified chirped optical pulses. Opt. Commun. 1985, 56, 219. [CrossRef]

4. Nakajima, K.; Nakanishi, H.; Kawakubo, T.; Ogata, A.; Kitagawa, Y.; Shiraga, H; Kodama, R.; Zhang, T.; Suzuki, K.; Kato, Y.; et al. Laser Wakefield Accelerator experiments using 1 ps 30 TW Nd:glass laser. In Proceedings of the IEEE International Conference on Particle Accelerators, Washington, DC, USA, 17-20 May 1993; Volume 4, pp. 2556-2558. [CrossRef]

5. Nakajima, K.; Kawakubo, T.; Nakanishi, H.; Ogata, A.; Kato, Y.; Kitagawa, Y.; Kodama, R.; Mima, K.; Shiraga, H.; Suzuki, K.; et al. A proof-of-principle experiment of laser wakefield acceleration. Phys. Scr. 1994, T52, 61-64. [CrossRef]

6. Fourkal, E.; Shahine, B.; Ding, M.; Li, J.S.; Tajima, T.; Ma, C.M. Particle in cell simulation of laser-accelerated proton beams for radiation therapy. Med. Phys. 2002, 29, 2788-2798. [CrossRef]

7. Newhauser, W.; Zhang, R. The physics of proton therapy. Phys. Med. Biol. 2015, 60, R155. [CrossRef] [PubMed]

8. Bulanov, S.V.; Daido, H.; Esirkepov, T.Z.; Khoroshkov, V.S.; Koga, J.; Nishihara, K.; Pegoraro, F.; Tajima, T.; Yamagiwa, M. Feasibility of Using Laser Ion Accelerators in Proton Therapy. AIP Conf. Proc. 2004, 740, 414-429. [CrossRef]

9. Tajima, T. Prospect for compact medical laser accelerators. J. Jpn. Soc. Therap. Radiat. Oncol. 1997, 9, 83-85.

10. Chiu, C.; Fomytskyi, M.; Grigsby, F.; Raischel, F.; Downer, M.C.; Tajima, T. Laser electron accelerators for radiation medicine: A feasibility study. Med. Phys. 2004, 31, 2042-2052. [CrossRef]

11. Kainz, K.K.; Hogstrom, K.R.; Antolak, J.A.; Almond, P.R.; Bloch, C.D.; Chiu, C.; Fomytskyi, M.; Raischel, F.; Downer, M.; Tajima, T. Dose properties of a laser accelerated electron beam and prospects for clinical application. Med. Phys. 2004, 31, $2053-2067$. [CrossRef]

12. Giulietti, A.; Bourgeois, N.; Ceccotti, T.; Davoine, X.; Dobosz, S.; D'Oliveira, P.; Galimberti, M.; Galy, J.; Gamucci, A.; et al. Intense $\gamma$-Ray Source in the Giant-Dipole-Resonance Range Driven by 10-TW Laser Pulses. Phys. Rev. Lett. 2008, 101, 105002. [CrossRef]

13. Giulietti, A. (Ed.) Laser-Driven Particle Acceleration Towards Radiobiology and Medicine; Springer International Publishing: Cham, Switzerland, 2016.

14. Nakajima, K.; Yuan, J.; Chen, L.; Sheng, Z. Laser-driven very high energy electron/photon beam radiation therapy in conjunction with a robotic system. Appl. Sci. 2015, 5, 1-20. [CrossRef]

15. Nakajima, K. Laser-driven electron beam and radiation sources for basic, medical and industrial sciences. Proc. Jpn. Acad. Ser. B 2015, 91, 223-245. [CrossRef]

16. Mourou, G.; Brocklesby, W.; Tajima, T.; Limpert, J. The future is fibre accelerators. Nat. Photonics 2013, 7, 258. [CrossRef]

17. Mako, F.; Tajima, T. Collective ion acceleration by a reflexing electron beam: Model and scaling. Phys. Fluids 1984, 27, 1815-1820. [CrossRef]

18. Nicks, B.S.; Tajima, T.; Roa, D.; Nečas, A.; Mourou, G. Laser-wakefield application to oncology. Int. J. Mod. Phys. A 2019, 34, 1943016. [CrossRef]

19. Yao, Z.; Dekker, C.; Avouris, P., Electrical Transport Through Single-Wall Carbon Nanotubes. In Carbon Nanotubes: Synthesis, Structure, Properties, and Applications; Dresselhaus, M.S., Dresselhaus, G., Avouris, P., Eds.; Springer: Berlin/Heidelberg, Germany, 2001; pp. 147-171. [CrossRef]

20. Bandaru, P.R. Electrical properties and applications of carbon nanotube structures. J. Nanosci. Nanotechnol. 2007, 7, 1239. [CrossRef]

21. Li, Y.; Fahrenthold, E. Quantum conductance of copper-carbon nanotube composites. J. Eng. Mater. Technol. 2018, 140, 031007. [CrossRef]

22. Subramaniam, C.; Yamada, T.; Kobashi, K.; Sekiguchi, A.; Futaba, D.N.; Yumura, M.; Hata, K. One hundred fold increase in current carrying capacity in a carbon nanotube-copper composite. Nat. Commun. 2013, 4, 2202. [CrossRef]

23. Hong, S.; Myung, S. A flexible approach to mobility. Nat. Nanotechnol. 2007, 2, 207. [CrossRef]

24. Shiraishi, M.; Ata, M. Work function of carbon nanotubes. Carbon 2001, 39, 1913. [CrossRef]

25. Wheeler, Taborek, Chen, Dollar, and Shen]Zhang2016 Zhang, X.; Tajima, T.; Farinella, D.; Shin, Y.; Mourou, G.; Wheeler, J.; Taborek, P.; Chen, P.; Dollar, F.; Shen, B. Particle-in-cell simulation of x-ray wakefield acceleration and betatron radiation in nanotubes. Phys. Rev. Accel. Beams 2016, 19, 101004. [CrossRef]

26. Wilks, S.C.; Kruer, W.L.; Tabak, M.; Langdon, A.B. Absorption of ultra-intense laser pulses. Phys. Rev. Lett. 1992, 69, 1383-1386. [CrossRef] [PubMed]

27. Valenta, P.; Klimo, O.; Grittani, G.M.; Esirkepov, T.Z.; Korn, G.; Bulanov, S.V. Wakefield excited by ultrashort laser pulses in near-critical density plasmas. In Laser Acceleration of Electrons, Protons, and Ions V; Esarey, E., Schroeder, C.B., Schreiber, J., Eds.; International Society for Optics and Photonics (SPIE): London, UK, 2019; Volume 11037, pp. 57-65. [CrossRef]

28. O'Neil, T. Collisionless Damping of Nonlinear Plasma Oscillations. Phys. Fluids 1965, 8, 2255-2262. [CrossRef]

29. Lamb, H. Hydrodynamics; Dover Publications: New York, NY, USA, 1945; Chapter 8. 
30. Pukhov, A.; Meyer-ter-Vehn, J. Relativistic Magnetic Self-Channeling of Light in Near-Critical Plasma: Three-Dimensional Particle-in-Cell Simulation. Phys. Rev. Lett. 1996, 76, 3975-3978. [CrossRef]

31. Sylla, F.; Flacco, A.; Kahaly, S.; Veltcheva, M.; Lifschitz, A.; Malka, V.; d'Humières, E.; Andriyash, I.; Tikhonchuk, V. Short Intense Laser Pulse Collapse in Near-Critical Plasma. Phys. Rev. Lett. 2013, 110, 085001. [CrossRef]

32. Chen, H.; Wilks, S.C. Evidence of enhanced effective hot electron temperatures in ultraintense laser-solid interactions due to reflexing. Laser Part Beams 2005, 23, 411. [CrossRef]

33. Nakamura, T.; Mima, K. Magnetic-Dipole Vortex Generation by Propagation of Ultraintense and Ultrashort Laser Pulses in Moderate-Density Plasmas. Phys. Rev. Lett. 2008, 100, 205006. [CrossRef]

34. Snavely, R.A.; Key, M.H.; Hatchett, S.P.; Cowan, T.E.; Roth, M.; Phillips, T.W.; Stoyer, M.A.; Henry, E.A.; Sangster, T.C.; Singh, M.S.; et al. Intense High-Energy Proton Beams from Petawatt-Laser Irradiation of Solids. Phys. Rev. Lett. 2000, 85, 2945-2948. [CrossRef]

35. Esirkepov, T.; Borghesi, M.; Bulanov, S.V.; Mourou, G.; Tajima, T. Highly Efficient Relativistic-Ion Generation in the Laser-Piston Regime. Phys. Rev. Lett. 2004, 92, 175003. [CrossRef]

36. Tajima, T. Laser acceleration in novel media. Eur. Phys. J. Spec. Top. 2014, 223, 1037-1044. [CrossRef]

37. Myung, N.V.; Lim, J.; Fleurial, J.P.; Yun, M.; West, W.; Choi, D. Alumina nanotemplate fabrication on silicon substrate. Nanotechnology 2004, 15, 833-838. [CrossRef]

38. Rau, B.; Tajima, T.; Hojo, H. Coherent Electron Acceleration by Subcycle Laser Pulses. Phys. Rev. Lett. 1997, 78, 3310-3313. [CrossRef]

39. Hassan, M.T.; Luu, T.T.; Moulet, A.; Raskazovskaya, O.; Zhokhov, P.; Garg, M.; Karpowicz, N.; Zheltijov, A.M.; Pervak, V.; Krausz, F.; et al. Optical attosecond pulses and tracking the nonlinear response of bound electrons. Nature 2016, 530, 66. [CrossRef]

40. Esarey, E.; Schroeder, C.B.; Leemans, W.P. Physics of laser-driven plasma-based electron accelerators. Rev. Mod. Phys. 2009, 81, 1229-1285. [CrossRef]

41. Lau, C.K.; Yeh, P.C.; Luk, O.; McClenaghan, J.; Ebisuzaki, T.; Tajima, T. Ponderomotive acceleration by relativistic waves. Phys. Rev. Spec. Top.-Accel. Beams 2015, 18, 024401. [CrossRef]

42. Bionta, M.R.; Chalopin, B.; Masseboeuf, A.; Chatel, B. First results on laser-induced field emission from a CNT-based nanotip. Ultramicroscopy 2015, 159, 152. [CrossRef]

43. Adli, E.; Ahuja, A.; Apsimon, O.; Apsimon, R.; Bachmann, A.-M.; Barrientos, D.; Batsch, F.; Bauche, J.; Berglyd Olsen, V.K.; Bernardini, M.; et al. Acceleration of electrons in the plasma wakefield of a proton bunch. Nature 2018, 561, 363-367. [CrossRef]

44. Salehi, F.; Goers, A.J.; Hine, G.A.; Feder, L.; Kuk, D.; Miao, B.; Woodbury, D.; Kim, K.Y.; Milchberg, H.M. MeV electron acceleration at $1 \mathrm{kHz}$ with $<10 \mathrm{~mJ}$ laser pulses. Opt. Lett. 2017, 42, 215-218. [CrossRef]

45. Sharp, K.; Matschinsky, F. Translation of Ludwig Boltzmann's Paper “On the Relationship between the Second Fundamental Theorem of the Mechanical Theory of Heat and Probability Calculations Regarding the Conditions for Thermal Equilibrium" Sitzungberichte der Kaiserlichen Akademie der Wissenschaften. Mathematisch-Naturwissen Classe. Abt. II, LXXVI 1877, pp. 373-435 (Wien. Ber. 1877, 76:373-435). Reprinted in Wiss. Abhandlungen, Vol. II, reprint 42, pp. 164-223. Barth, Leipzig, 1909. Entropy 2015, 17, 1971-2009. [CrossRef]

46. Hu, R.; Lu, H.; Shou, Y.; Lin, C.; Zhuo, H.; Chen, C.; Yan, X. Brilliant GeV electron beam with narrow energy spread generated by a laser plasma accelerator. Phys. Rev. Accel. Beams 2016, 19, 091301. [CrossRef]

47. Döpp, A.; Thaury, C.; Guillaume, E.; Massimo, F.; Lifschitz, A.; Andriyash, I.; Goddet, J.P.; Tazfi, A.; Ta Phuoc, K.; Malka, V. Energy-Chirp Compensation in a Laser Wakefield Accelerator. Phys. Rev. Lett. 2018, 121, 074802. [CrossRef] 